\title{
On the right spread ordering of series systems with two heterogeneous Weibull components
}

\author{
Longxiang Fang* and Wei Tang
}

"Correspondence:

Ixfang@fudan.edu.cn

College of Mathematical and

Computer Science, Anhui Normal

University, Wuhu, 241002, China

\begin{abstract}
In this paper, we discuss the variability ordering of lifetimes of series systems with two independent heterogeneous Weibull components in terms of the right spread order. We give some sufficient conditions implying the right spread order between lifetimes of series systems.
\end{abstract}

MSC: Primary 60E15; 62G30

Keywords: Weibull distribution; series systems; right spread order; majorization

\section{Introduction}

Order statistics play an important role in statistical inference, life testings, reliability theory and many other areas. Let $X_{1}, \ldots, X_{n}$ be $n$ random variables and let $X_{i: n}$ denotes their $i$ th order statistic, $i=1, \ldots, n$. In reliability theory, the $k$ th order statistic $X_{k: n}$ corresponds to the lifetime of a $(n-k+1)$-out-of- $n$ system. Parallel and series systems are the building blocks of more complex coherent systems, wherein the lifetime of a series system corresponds to the smallest order statistic $X_{1: n}$ and the lifetime of a parallel system corresponds to the largest order statistic $X_{n: n}$. Misra and Misra [1] have considered the stochastic comparisons of series systems according to the reversed hazard rate order and the likelihood ratio order. Misra et al. [2] have compared lifetimes of series (parallel) systems arising out of different allocations of one or two standby redundancies in light of the hazard rate order, the increasing concave (convex) order, and the stochastic precedence order. Moreover, other authors have studied stochastic comparisons of lifetimes of series and parallel systems; for example, see [3-12], and references cited therein.

To continue our discussion, we need definitions of some stochastic orders and the concept of majorization which is given in Section 2. Zhao and Balakrishnan [4] have focused on comparison the largest order statistic in terms of the important right spread order from two heterogeneous exponential variables, that is, the lifetime of a parallel system with two independent heterogeneous exponential components. Let $X_{1}, X_{2}$ be independent exponential random variables with $X_{i}$ having hazard rate $\lambda_{i}, i=1,2$. Let $X_{1}^{*}, X_{2}^{*}$ be another set of independent exponential random variables with $X_{i}^{*}$ having hazard rate $\lambda_{i}^{*}, i=1,2$. Zhao and Balakrishnan [4] showed that $X_{2: 2}^{*} \leq_{R S} X_{2: 2}$ under the condition

$$
\lambda_{1} \leq \lambda_{1}^{*} \leq \lambda_{2}^{*} \leq \lambda_{2} \quad \text { and } \quad \frac{1}{\lambda_{1}}+\frac{1}{\lambda_{2}} \geq \frac{1}{\lambda_{1}^{*}}+\frac{1}{\lambda_{2}^{*}} .
$$

○2014 Fang and Tang; licensee Springer. This is an Open Access article distributed under the terms of the Creative Commons Attribution License (http://creativecommons.org/licenses/by/2.0), which permits unrestricted use, distribution, and reproduction in any medium, provided the original work is properly cited. 
Weibull distribution is commonly used lifetime distribution in modeling lifetime data. Let $X$ be a Weibull random variable with shape parameter $\alpha$ and scale parameter $\lambda$, and with probability density function

$$
h(x, \alpha, \lambda)=\alpha x^{\alpha-1} \lambda^{\alpha} e^{-(\lambda x)^{\alpha}}, \quad x>0, \alpha>0, \lambda>0 .
$$

Recently, Prabhakar Murthy et al. [13] have studied Weibull models in detail. The exponential distribution and the Rayleigh distribution can be obtained from Weibull distribution by putting $\alpha=1$ and $\alpha=2$, respectively.

Let $\left(X_{1}, \ldots, X_{n}\right)$ be a vector of independent Weibull random variables with common shape parameter $\alpha>0$ and respective scale parameters $\lambda_{i}, i=1, \ldots, n$. Let $\left(Y_{1}, \ldots, Y_{n}\right)$ be another vector of independent Weibull random variables with common shape parameter $\alpha$ and scale parameter $\lambda$. When $\lambda=\left(\prod_{i=1}^{n} \lambda_{i}\right)^{\frac{1}{n}}$, Khaledi and Kochar [6] proved that $Y_{n: n} \leq_{\text {disp }}$ $X_{n: n}$ for $0<\alpha \leq 1$. Let $\lambda_{\min }$ denote the minimum of $\lambda_{i}, i=1, \ldots, n$. When $\lambda \geq \frac{\left(\prod_{i=1}^{n} \lambda_{i}^{\alpha} \frac{1}{n}\right.}{\lambda_{\min }^{\alpha-1}}$, Fang and Zhang [12] proved that $Y_{n: n} \leq_{\text {disp }} X_{n: n}$ for $\alpha>1$. Fang and Zhang [11] showed that $Y_{1: n} \leq_{c} X_{1: n}$; and if $\lambda \geq\left(\frac{1}{n} \sum_{i=1}^{n} \lambda_{i}^{\alpha}\right)^{\frac{1}{\alpha}}$, then $Y_{1: n} \leq_{\text {disp }} X_{1: n}$; and if $\lambda=\left(\prod_{i=1}^{n} \lambda_{i}\right)^{\frac{1}{n}}$, then $X_{1: n} \leq_{r h} Y_{1: n}$. Let $X_{1}^{*}, \ldots, X_{n}^{*}$ be independent random variables with common shape parameter $\alpha>0$ and respective scale parameters $\lambda_{i}^{*}, i=1, \ldots, n$. Fang and Zhang [11] showed that $\left(\lambda_{1}^{*}, \ldots, \lambda_{n}^{*}\right) \leq_{m}\left(\lambda_{1}, \ldots, \lambda_{n}\right)$ implies that $X_{1: n} \leq_{r h} X_{1: n}^{*}$ for $\alpha>1$ and $X_{1: n}^{*} \leq_{r h} X_{1: n}$ for $0<\alpha \leq 1$. In this paper, we compare the lifetimes of series systems with respect to the right spread order arising from two independent heterogeneous Weibull random variables. Let $\left(X_{1}, X_{2}\right)$ be a vector of independent Weibull random variables with common shape parameter $\alpha>0$ and respective scale parameters $\lambda_{1}$ and $\lambda_{2}$, and let $\left(X_{1}^{*}, X_{2}^{*}\right)$ be another vector of independent Weibull random variables with common shape parameter $\alpha$ and respective scale parameters $\lambda_{1}^{*}$ and $\lambda_{2}^{*}$. Under the condition $\left(\lambda_{1}, \lambda_{2}\right) \leq_{m}\left(\lambda_{1}^{*}, \lambda_{2}^{*}\right)$, we will show that if $0<\alpha \leq 1$ then $X_{1: 2} \leq_{R S} X_{1: 2}^{*}$; and if $\alpha>1$ then $X_{1: 2}^{*} \leq_{R S} X_{1: 2}$. On the other hand, under the condition

$$
\min \left(\lambda_{1}^{*}, \lambda_{2}^{*}\right) \leq \min \left(\lambda_{1}, \lambda_{2}\right) \leq \max \left(\lambda_{1}, \lambda_{2}\right) \leq \max \left(\lambda_{1}^{*}, \lambda_{2}^{*}\right) \quad \text { and } \quad \frac{1}{\lambda_{1}}+\frac{1}{\lambda_{2}} \geq \frac{1}{\lambda_{1}^{*}}+\frac{1}{\lambda_{2}^{*}} ;
$$

or

$$
\min \left(\lambda_{1}, \lambda_{2}\right) \leq \min \left(\lambda_{1}^{*}, \lambda_{2}^{*}\right) \leq \max \left(\lambda_{1}, \lambda_{2}\right) \leq \max \left(\lambda_{1}^{*}, \lambda_{2}^{*}\right)
$$

we establish $X_{1: 2}^{*} \leq_{R S} X_{1: 2}$.

\section{Preliminaries}

Suppose that the random variables $X$ and $Y$ have distribution functions $F(x)$ and $G(x)$, survival functions $\bar{F}(x)$ and $\bar{G}(x)$, the right continuous inverse functions $F^{-1}(x)$ and $G^{-1}(x)$, density functions $f(x)$ and $g(x)$, and the reversed hazard rate functions $\tilde{r}_{X}(x)=\frac{f(x)}{F(x)}$ and $\tilde{r}_{Y}(x)=\frac{g(x)}{G(x)}$, respectively. In this paper, all the random variables are assumed to be nonnegative and having support $[0,+\infty)$. Let $R=(-\infty,+\infty)$.

Throughout this paper, the notions increasing and decreasing mean nondecreasing and nonincreasing, respectively. 
Definition 2.1 $X$ is said to be

(i) less dispersive than $Y$ if

$$
F^{-1}(\beta)-F^{-1}(\alpha) \leq G^{-1}(\beta)-G^{-1}(\alpha)
$$

for $0<\alpha \leq \beta<1$, in symbols, $X \leq \operatorname{disp} Y$;

(ii) less right spread than $Y$ if

$$
\int_{F^{-1}(p)}^{\infty} \bar{F}(t) d t \leq \int_{G^{-1}(p)}^{\infty} \bar{G}(t) d t
$$

for $0 \leq p \leq 1$, in symbols, $X \leq_{R S} Y$;

(iii) smaller than $Y$ in the reversed hazard rate order if $\tilde{r}_{X}(x) \leq \tilde{r}_{Y}(x)$ for all $x>0$, or equivalently, if $\frac{F(x)}{G(x)}$ is decreasing in $x>0$; in symbols, $X \leq_{r h} Y$;

(iv) smaller than $Y$ in the convex transform order if $G^{-1} F(x)$ is convex in $x$ on the support of $X$, in symbols, $X \leq_{c} Y$.

The dispersive order and the right spread order are two basic partial orders comparing the variabilities in the two distributions. The right spread order has been widely used in many fields, especially in economics, insurance, and reliability theory. It is well known that

$$
X \leq_{\text {disp }} Y \quad \Rightarrow \quad X \leq_{R S} Y \quad \Rightarrow \quad \operatorname{Var}(X) \leq \operatorname{Var}(Y)
$$

Several notions of stochastic orders of varying degree of strength have been discussed in detail in [14].

Majorization is a very interesting topic in statistics, which is a pre-ordering on vectors by sorting all components in increasing order.

Definition 2.2 Let $\lambda=\left(\lambda_{1}, \lambda_{2}, \ldots, \lambda_{n}\right), \lambda^{*}=\left(\lambda_{1}^{*}, \lambda_{2}^{*}, \ldots, \lambda_{n}^{*}\right)$ denote two $n$-dimensional real vectors. Let $\lambda_{(1)} \leq \lambda_{(2)} \leq \cdots \leq \lambda_{(n)}, \lambda_{(1)}^{*} \leq \lambda_{(2)}^{*} \leq \cdots \leq \lambda_{(n)}^{*}$ be their ordered components. $\lambda^{*}$ is said to be majorized by $\lambda$, in symbols $\lambda^{*} \leq_{m} \lambda$, if $\sum_{i=1}^{j} \lambda_{(i)} \leq \sum_{i=1}^{j} \lambda_{(i)}^{*}$; for $j=1,2$, $\ldots, n-1$, and $\sum_{i=1}^{n} \lambda_{i}=\sum_{i=1}^{n} \lambda_{i}^{*}$.

For more details on majorization and their applications, one may refer to Marshall and Olkin [15].

The following two useful results, which established an equivalent characterization of dispersive order and the right spread order in one parameter family, will be needed to prove our main results.

Lemma 2.3 (Saunders and Moran [16]) Let $\left\{F_{a} \mid a \in R\right\}$ be a class of distribution functions, such that $F_{a}$ is supported on some interval $\left(x_{-}^{(a)}, x_{+}^{(a)}\right) \subseteq(0,+\infty)$ and has a density $f_{a}$ which does not vanish on any subinterval of $\left(x_{-}^{(a)}, x_{+}^{(a)}\right)$, where $x_{-}^{(a)}$ and $x_{+}^{(a)}$ denote the left and right end points, respectively. Then $F_{a} \leq \operatorname{disp} F_{a^{*}}, a, a^{*} \in R, a \leq a^{*}$, if and only if $\frac{F_{a}^{\prime}(x)}{f_{a}(x)}$ is decreasing in $x$, where $F_{a}^{\prime}(x)$ is the derivative of $F_{a}$ with respect to $a$.

Lemma 2.4 Let $\left\{F_{a} \mid a \in R\right\}$ be assumed as Lemma 2.3. Then

(i) $F_{a} \leq_{R S} F_{a^{*}}, a, a^{*} \in R, a \leq a^{*}$, if and only if $\frac{W_{a}^{\prime}(x)}{\bar{F}_{a}(x)}$ is increasing in $x$; 
(ii) $F_{a^{*}} \leq_{R S} F_{a}, a, a^{*} \in R, a \leq a^{*}$, if and only if $\frac{W_{a}^{\prime}(x)}{\bar{F}_{a}(x)}$ is decreasing in $x$; where $W_{a}^{\prime}(x)$ is the derivative of $W_{a}(x)=\int_{x}^{\infty} \bar{F}_{a}(t) d t$ with respect to $a$.

The proof of this lemma is similar to the proof of Lemma 3.1 of Kochar and Xu [17], so we omit it here.

\section{Main results}

Firstly, we consider stochastic comparison on the lifetimes of series systems arising from two independent heterogeneous Weibull random variables in terms of dispersive order.

Theorem 3.1 Let $\left(X_{1}, X_{2}\right)$ be a vector of independent Weibull random variables with common shape parameter $\alpha>0$ and respective scale parameters $\lambda$ and $\lambda_{1}$, and let $\left(Y_{1}, Y_{2}\right)$ be another vector of independent Weibull random variables with common shape parameter $\alpha$ and respective scale parameters $\lambda$ and $\lambda_{2}$. Suppose that $\lambda \geq \max \left(\lambda_{1}, \lambda_{2}\right)$. If $\lambda_{1} \leq \lambda_{2}$, then $Y_{1: 2} \leq_{\operatorname{disp}} X_{1: 2}$.

Proof Suppose $\lambda_{1} \leq \lambda_{2}$. Denote $a=\lambda-\lambda_{1}$ and $a^{*}=\lambda-\lambda_{2}$, we have $a \geq a^{*}$. The distribution function of $X_{1: 2}$, denoted by $F_{a}$, is given by

$$
F_{a}(x)=P\left(X_{1: 2} \leq x\right)=1-e^{-\left[\lambda^{\alpha}+(\lambda-a)^{\alpha}\right] x^{\alpha}}, \quad \text { for } x \geq 0
$$

Taking the derivative with respect to $a$, we have

$$
F_{a}^{\prime}(x)=-\alpha(\lambda-a)^{\alpha-1} x^{\alpha} e^{-\left[\lambda^{\alpha}+(\lambda-a)^{\alpha}\right] x^{\alpha}}
$$

Taking the derivative with respect to $x$, we obtain the density function of $F_{a}(x)$ as

$$
f_{a}(x)=\alpha x^{\alpha-1}\left[\lambda^{\alpha}+(\lambda-a)^{\alpha}\right] e^{-\left[\lambda^{\alpha}+(\lambda-a)^{\alpha}\right] x^{\alpha}} .
$$

From (3.1) and (3.2), we see that

$$
\frac{F_{a}^{\prime}(x)}{f_{a}(x)}=-\frac{(\lambda-a)^{\alpha-1} x}{\lambda^{\alpha}+(\lambda-a)^{\alpha}}
$$

is decreasing in $x \geq 0$. From Lemma 2.3, we can conclude that $Y_{1: 2} \leq_{\text {disp }} X_{1: 2}$.

Next, we provide some sufficient conditions on the lifetimes of series systems arising from two independent heterogeneous Weibull random variables in terms of the right spread order.

Theorem 3.2 Let $\left(X_{1}, X_{2}\right)$ be a vector of independent Weibull random variables with common shape parameter $\alpha>0$ and respective scale parameters $\lambda_{1}$ and $\lambda_{2}$, and let $\left(X_{1}^{*}, X_{2}^{*}\right)$ be another vector of independent Weibull random variables with common shape parameter $\alpha$ and respective scale parameters $\lambda_{1}^{*}$ and $\lambda_{2}^{*}$. Suppose that $\left(\lambda_{1}, \lambda_{2}\right) \leq_{m}\left(\lambda_{1}^{*}, \lambda_{2}^{*}\right)$.

(i) If $0<\alpha \leq 1$, then $X_{1: 2} \leq_{R S} X_{1: 2}^{*}$;

(ii) if $\alpha>1$, then $X_{1: 2}^{*} \leq_{R S} X_{1: 2}$. 
Proof Without loss of generality, let us assume that $\lambda_{1} \leq \lambda_{2}$ and $\lambda_{1}^{*} \leq \lambda_{2}^{*}$. Since $\left(\lambda_{1}, \lambda_{2}\right) \leq_{m}$ $\left(\lambda_{1}^{*}, \lambda_{2}^{*}\right)$, we have $\lambda_{1}^{*} \leq \lambda_{1}$ and $\lambda_{1}+\lambda_{2}=\lambda_{1}^{*}+\lambda_{2}^{*}$. Let $\lambda_{1}=\lambda, \lambda_{1}^{*}=\lambda^{*}, \lambda_{1}+\lambda_{2}=\lambda_{1}^{*}+\lambda_{2}^{*}=a$. We then have $\lambda^{*} \leq \lambda, \lambda_{2}=a-\lambda, \lambda_{2}^{*}=a-\lambda^{*}$.

The survival function of $X_{1: 2}$, denoted by $\bar{F}_{\lambda}$, can be expressed as

$$
\bar{F}_{\lambda}(x)=P\left(X_{1: 2}>x\right)=e^{-\left[\lambda^{\alpha}+(a-\lambda)^{\alpha}\right] x^{\alpha}}, \quad \text { for } x \geq 0 .
$$

Let

$$
W_{\lambda}(x)=\int_{x}^{\infty} \bar{F}_{\lambda}(t) d t=\int_{x}^{\infty} e^{-\left[\lambda^{\alpha}+(a-\lambda)^{\alpha}\right] t^{\alpha}} d t
$$

Taking the derivative with respect to $\lambda$, we have

$$
W_{\lambda}^{\prime}(x)=\alpha\left[(a-\lambda)^{\alpha-1}-\lambda^{\alpha-1}\right] \int_{x}^{\infty} t^{\alpha} e^{-\left[\lambda^{\alpha}+(a-\lambda)^{\alpha}\right] t^{\alpha}} d t .
$$

So, using (3.3) and (3.4), we obtain

$$
\begin{aligned}
\frac{W_{\lambda}^{\prime}(x)}{\bar{F}_{\lambda}(x)} & =\frac{\alpha\left[(a-\lambda)^{\alpha-1}-\lambda^{\alpha-1}\right] \int_{x}^{\infty} t^{\alpha} e^{-\left[\lambda^{\alpha}+(a-\lambda)^{\alpha}\right] t^{\alpha}} d t}{e^{-\left[\lambda^{\alpha}+(a-\lambda)^{\alpha}\right] x^{\alpha}}} \\
& =\frac{(a-\lambda)^{\alpha-1}-\lambda^{\alpha-1}}{(a-\lambda)^{\alpha}+\lambda^{\alpha}} \cdot \frac{\int_{x}^{\infty} t^{\alpha} e^{-\left[\lambda^{\alpha}+(a-\lambda)^{\alpha}\right] t^{\alpha}} d t}{\int_{x}^{\infty} t^{\alpha-1} e^{-\left[\lambda^{\alpha}+(a-\lambda)^{\alpha}\right] t^{\alpha}} d t} \\
& =\frac{(a-\lambda)^{\alpha-1}-\lambda^{\alpha-1}}{(a-\lambda)^{\alpha}+\lambda^{\alpha}} m(x),
\end{aligned}
$$

where

$$
m(x)=\frac{\int_{x}^{\infty} t^{\alpha} e^{-\left[\lambda^{\alpha}+(a-\lambda)^{\alpha}\right] t^{\alpha}} d t}{\int_{x}^{\infty} t^{\alpha-1} e^{-\left[\lambda^{\alpha}+(a-\lambda)^{\alpha}\right] t^{\alpha}} d t}, \quad x \geq 0 .
$$

By taking the derivative with respect to $x$, we have

$$
\begin{aligned}
m^{\prime}(x) & =\frac{x^{\alpha-1} e^{-\left[\lambda^{\alpha}+(a-\lambda)^{\alpha}\right] x^{\alpha}}\left(\int_{x}^{\infty} t^{\alpha} e^{-\left[\lambda^{\alpha}+(a-\lambda)^{\alpha}\right] t^{\alpha}} d t-x \int_{x}^{\infty} t^{\alpha-1} e^{-\left[\lambda^{\alpha}+(a-\lambda)^{\alpha}\right] t^{\alpha}} d t\right)}{\left(\int_{x}^{\infty} t^{\alpha-1} e^{-\left[\lambda^{\alpha}+(a-\lambda)^{\alpha}\right] t^{\alpha}} d t\right)^{2}} \\
& =\frac{x^{\alpha-1} e^{-\left[\lambda^{\alpha}+(a-\lambda)^{\alpha}\right] x^{\alpha}} \int_{x}^{\infty} t^{\alpha-1}(t-x) e^{-\left[\lambda^{\alpha}+(a-\lambda)^{\alpha}\right] t^{\alpha}} d t}{\left(\int_{x}^{\infty} t^{\alpha-1} e^{-\left[\lambda^{\alpha}+(a-\lambda)^{\alpha} t^{\alpha}\right.} d t\right)^{2}} \\
& \geq 0, \quad \forall x \geq 0 .
\end{aligned}
$$

Thus, $m(x)$ is increasing in $x \geq 0$.

Since $0<\alpha \leq 1$, then $(a-\lambda)^{\alpha-1}-\lambda^{\alpha-1} \leq 0$, which implies $\frac{W_{\lambda}^{\prime}(x)}{\bar{F}_{\lambda}(x)}$ is decreasing in $x \geq 0$. On the other hand, if $\alpha>1$, then $(a-\lambda)^{\alpha-1}-\lambda^{\alpha-1}>0$, which implies $\frac{W_{\lambda}^{\prime}(x)}{\bar{F}_{\lambda}(x)}$ is increasing in $x \geq 0$. Now the proof follows from Lemma 2.4.

Theorem 3.3 Let $\left(X_{1}, X_{2}\right)$ be a vector of independent Weibull random variables with common shape parameter $\alpha>0$ and respective scale parameters $\lambda_{1}$ and $\lambda_{2}$, and let $\left(X_{1}^{*}, X_{2}^{*}\right)$ be another vector of independent Weibull random variables with common shape parameter 
$\alpha$ and respective scale parameters $\lambda_{1}^{*}$ and $\lambda_{2}^{*}$. Suppose that $\max \left(\lambda_{1}, \lambda_{2}\right) \leq \max \left(\lambda_{1}^{*}, \lambda_{2}^{*}\right)$ and $\frac{1}{\lambda_{1}}+\frac{1}{\lambda_{2}}=\frac{1}{\lambda_{1}^{*}}+\frac{1}{\lambda_{2}^{*}}$, then $X_{1: 2}^{*} \leq_{R S} X_{1: 2}$.

Proof Without loss of generality, we assume that $\lambda_{1} \leq \lambda_{2}$ and $\lambda_{1}^{*} \leq \lambda_{2}^{*}$. Let $\lambda_{1}=\lambda, \lambda_{1}^{*}=\lambda^{*}$, $\frac{1}{\lambda_{1}}+\frac{1}{\lambda_{2}}=\frac{1}{\lambda_{1}^{*}}+\frac{1}{\lambda_{2}^{*}}=a$. We then have $\lambda_{2}=\frac{1}{a-\frac{1}{\lambda}}, \lambda_{2}^{*}=\frac{1}{a-\frac{1}{\lambda^{*}}}$, and $\frac{1}{a}<\lambda^{*} \leq \lambda$.

$\bar{F}_{\lambda}(x)$, the survival function of $X_{1: 2}$, can be expressed as

$$
\bar{F}_{\lambda}(x)=P\left(X_{1: 2}>x\right)=e^{-\left[\lambda^{\alpha}+\frac{1}{\left(a-\frac{1}{\lambda}\right)^{\alpha}}\right] x^{\alpha}}, \quad \text { for } x \geq 0
$$

So, we obtain

$$
W_{\lambda}(x)=\int_{x}^{\infty} \bar{F}_{\lambda}(t) d t=\int_{x}^{\infty} e^{-\left[\lambda^{\alpha}+\frac{1}{\left(a-\frac{1}{\lambda}\right)^{\alpha}}\right] t^{\alpha}} d t
$$

Taking the derivative with respect to $\lambda$, we have

$$
W_{\lambda}^{\prime}(x)=\alpha \lambda^{\alpha-1}\left[\frac{1}{(a \lambda-1)^{\alpha+1}}-1\right] \int_{x}^{\infty} t^{\alpha} e^{-\left[\lambda^{\alpha}+\frac{1}{\left(a-\frac{1}{\lambda}\right)^{\alpha}}\right] t^{\alpha}} d t
$$

By (3.5) and (3.6), we obtain

$$
\begin{aligned}
\frac{W_{\lambda}^{\prime}(x)}{\bar{F}_{\lambda}(x)} & =\frac{\alpha \lambda^{\alpha-1}\left[\frac{1}{(a \lambda-1)^{\alpha+1}}-1\right] \int_{x}^{\infty} t^{\alpha} e^{-\left[\lambda^{\alpha}+\frac{1}{\left(a-\frac{1}{\lambda}\right)^{\alpha}}\right] t^{\alpha}} d t}{e^{-\left[\lambda \alpha+\frac{1}{\left(a-\frac{1}{\lambda}\right)^{\alpha}}\right] x^{\alpha}}} \\
& =\frac{\lambda^{\alpha-1}\left[\frac{1}{(a \lambda-1)^{\alpha+1}}-1\right]}{\lambda^{\alpha}+\frac{1}{\left(a-\frac{1}{\lambda}\right)^{\alpha}}} \cdot \frac{\int_{x}^{\infty} t^{\alpha} e^{-\left[\lambda^{\alpha}+\frac{1}{\left(a-\frac{1}{\lambda}\right)^{\alpha}}\right] t^{\alpha}} d t}{\int_{x}^{\infty} t^{\alpha-1} e^{-\left[\lambda^{\alpha}+\frac{1}{\left(a-\frac{1}{\lambda}\right)^{\alpha}}\right] t^{\alpha}} d t} \\
& =\frac{\lambda^{\alpha-1}\left[\frac{1}{(a \lambda-1)^{\alpha+1}}-1\right]}{\lambda^{\alpha}+\frac{1}{\left(a-\frac{1}{\lambda}\right)^{\alpha}}} n(x),
\end{aligned}
$$

where

$$
n(x)=\frac{\int_{x}^{\infty} t^{\alpha} e^{-\left[\lambda^{\alpha}+\frac{1}{\left(a-\frac{1}{\lambda}\right)^{\alpha}}\right] t^{\alpha}} d t}{\int_{x}^{\infty} t^{\alpha-1} e^{-\left[\lambda^{\alpha}+\frac{1}{\left(a-\frac{1}{\lambda}\right)^{\alpha}}\right] t^{\alpha}} d t}, \quad x \geq 0
$$

By taking the derivative with respect to $x$, we have

$$
\begin{aligned}
n^{\prime}(x) & =\frac{x^{\alpha-1} e^{-\left[\lambda^{\alpha}+\frac{1}{\left(a-\frac{1}{\lambda}\right)^{\alpha}}\right] x^{\alpha}} \int_{x}^{\infty} t^{\alpha-1}(t-x) e^{-\left[\lambda^{\alpha}+\frac{1}{\left(a-\frac{1}{\lambda}\right)^{\alpha}}\right] t^{\alpha}} d t}{\left(\int_{x}^{\infty} t^{\alpha-1} e^{-\left[\lambda^{\alpha}+\frac{1}{\left(a-\frac{1}{\lambda}\right)^{\alpha}}\right] t^{\alpha}} d t\right)^{2}} \\
& \geq 0, \quad \forall x \geq 0 .
\end{aligned}
$$

Thus, $n(x)$ is increasing in $x \geq 0$.

Since $\frac{1}{\lambda}+\frac{1}{\lambda_{2}}=a$ and $\lambda \leq \lambda_{2}$, it is easy to see that $\frac{1}{\lambda} \geq \frac{a}{2}$, hence $a \lambda-1 \leq 1$, implying $\frac{1}{(a \lambda-1)^{\alpha+1}}-1 \geq 0$. Therefore, $\frac{W_{\lambda}^{\prime}(x)}{\bar{F}_{\lambda}(x)}$ is increasing in $x \geq 0$. So, the theorem follows from Lemma 2.4(i). 
Theorem 3.4 Let $\left(X_{1}, X_{2}\right)$ be a vector of independent Weibull random variables with common shape parameter $\alpha>0$ and respective scale parameters $\lambda_{1}$ and $\lambda_{2}$, and let $\left(X_{1}^{*}, X_{2}^{*}\right)$ be another vector of independent Weibull random variables with common shape parameter $\alpha$ and respective scale parameters $\lambda_{1}^{*}$ and $\lambda_{2}^{*}$. Suppose that

(i) $\min \left(\lambda_{1}^{*}, \lambda_{2}^{*}\right) \leq \min \left(\lambda_{1}, \lambda_{2}\right) \leq \max \left(\lambda_{1}, \lambda_{2}\right) \leq \max \left(\lambda_{1}^{*}, \lambda_{2}^{*}\right)$ and $\frac{1}{\lambda_{1}}+\frac{1}{\lambda_{2}} \geq \frac{1}{\lambda_{1}^{*}}+\frac{1}{\lambda_{2}^{*}}$; or

(ii) $\min \left(\lambda_{1}, \lambda_{2}\right) \leq \min \left(\lambda_{1}^{*}, \lambda_{2}^{*}\right) \leq \max \left(\lambda_{1}, \lambda_{2}\right) \leq \max \left(\lambda_{1}^{*}, \lambda_{2}^{*}\right)$.

Then $X_{1: 2}^{*} \leq_{R S} X_{1: 2}$.

Proof Without loss of generality, let us assume that $\lambda_{1} \leq \lambda_{2}$ and $\lambda_{1}^{*} \leq \lambda_{2}^{*}$. Then Assumptions (i) and (ii) provide

$$
\lambda_{1}^{*} \leq \lambda_{1} \leq \lambda_{2} \leq \lambda_{2}^{*} \quad \text { and } \quad \frac{1}{\lambda_{1}}+\frac{1}{\lambda_{2}} \geq \frac{1}{\lambda_{1}^{*}}+\frac{1}{\lambda_{2}^{*}},
$$

and

$$
\lambda_{1} \leq \lambda_{1}^{*} \leq \lambda_{2} \leq \lambda_{2}^{*} \text { and } \frac{1}{\lambda_{1}}+\frac{1}{\lambda_{2}} \geq \frac{1}{\lambda_{1}^{*}}+\frac{1}{\lambda_{2}^{*}},
$$

respectively.

So, the results for the case when $\frac{1}{\lambda_{1}}+\frac{1}{\lambda_{2}}=\frac{1}{\lambda_{1}^{*}}+\frac{1}{\lambda_{2}^{*}}$ can be obtained immediately from Theorem 3.3. In what follows, we consider that $\frac{1}{\lambda_{1}}+\frac{1}{\lambda_{2}}>\frac{1}{\lambda_{1}^{*}}+\frac{1}{\lambda_{2}^{*}}$. Let $\lambda^{\prime}=\frac{1}{\frac{1}{\lambda_{1}}+\frac{1}{\lambda_{2}}-\frac{1}{\lambda_{2}^{*}}}$, then

$$
\frac{1}{\lambda_{1}}+\frac{1}{\lambda_{2}}=\frac{1}{\lambda^{\prime}}+\frac{1}{\lambda_{2}^{*}} \text { and } \quad \lambda^{\prime}<\lambda_{1}^{*}
$$

Let $\left(Y_{1}, Y_{2}\right)$ be a vector of independent Weibull random variables with common shape parameter $\alpha$ and respective scale parameters $\lambda^{\prime}$ and $\lambda_{2}^{*}$. From Theorem 3.3, it follows that $Y_{1: 2} \leq_{R S} X_{1: 2}$. In addition, we have $X_{1: 2}^{*} \leq_{\text {disp }} Y_{1: 2}$ using Theorem 3.1 which further implies $X_{1: 2}^{*} \leq_{R S} Y_{1: 2}$. Thus we have $X_{1: 2}^{*} \leq_{R S} X_{1: 2}$.

Finally, we present some applications of the results established above. In reliability analysis, we all know the lifetime of a $k$-out-of- $n$ system can be represented as $X_{n-k+1: n}$. Reliability engineers are not only interested in controlling the mean lifetime of a system, but also comparing the variability of the lifetimes of two systems. The lifetime of a series system corresponds to the smallest order statistic $X_{1: n}$. Misra and Misra [1] have compared the lifetimes of series systems according to the reversed hazard rate order and the likelihood ratio order. We have considered the lifetimes of series systems with two independent heterogeneous Weibull components in terms of the right spread order. The results lead to the following insight with regard to $n$-out-of- $n$ systems.

Example 3.5 One $n$-out-of- $n$ system $A$ with independent heterogeneous Weibull components $\left(X_{1}, X_{2}\right)$, which have common shape parameter $\alpha>0$ and scale parameter vector $\left(\lambda_{1}, \lambda_{2}\right)$, the other $n$-out-of- $n$ system $B$ with independent heterogeneous Weibull components $\left(X_{1}^{*}, X_{2}^{*}\right)$, which have common shape parameter $\alpha>0$ and scale parameter vector $\left(\lambda_{1}^{*}, \lambda_{2}^{*}\right)$.

(a) Suppose that $\left(\lambda_{1}, \lambda_{2}\right)=(3,2),\left(\lambda_{1}^{*}, \lambda_{2}^{*}\right)=(1,4)$. If $\alpha=0.4$, then the system $A$ has a smaller variability than $B$ by Theorem 3.2(i); if $\alpha=2$, then the system $B$ has a smaller variability than $A$ by Theorem 3.2(ii). 
(b) Suppose that $\left(\lambda_{1}, \lambda_{2}\right)=(4,5),\left(\lambda_{1}^{*}, \lambda_{2}^{*}\right)=(6,60 / 17)$. Then the system $B$ has a smaller variability than $A$ by Theorem 3.3 .

(c) Suppose that $\left(\lambda_{1}, \lambda_{2}\right)=(2,4),\left(\lambda_{1}^{*}, \lambda_{2}^{*}\right)=(1.9,5)$. Then the system $B$ has a smaller variability than $A$ by Theorem 3.4(i).

(d) Suppose that $\left(\lambda_{1}, \lambda_{2}\right)=(4,2),\left(\lambda_{1}^{*}, \lambda_{2}^{*}\right)=(2.1,5)$. Then the system $B$ has a smaller variability than $A$ by Theorem 3.4(ii).

\section{Competing interests}

The authors declare that they have no competing interests.

\section{Authors' contributions}

The main idea and writing of this paper were carried out by LF. WT performed all the steps of the proofs in this research. All authors read and approved the final manuscript.

\section{Acknowledgements}

This research is supported by the National Natural Science Foundation of China (No. 11071045, 11201003); the Nationa Statistical Science Research Project of China (No. 2012LY158); the Provincial Natural Science Research Project of Anhui Colleges (No. KJ2013A137); the National Natural Science Foundation of Anhui Province (No. 1408085MA07).

Received: 23 September 2013 Accepted: 28 April 2014 Published: 13 May 2014

\section{References}

1. Misra, N, Misra, AK: New results on stochastic comparisons of two-component series and parallel systems. Stat. Probab. Lett. 82, 283-290 (2012)

2. Misra, N, Misra, AK, Dhariyal, ID: Standby redundancy allocations in series and parallel systems. J. Appl. Probab. 48 43-55 (2011)

3. Zhao, P, Balakrishnan, N: New results on comparisons of parallel systems with heterogeneous gamma components. Stat. Probab. Lett. 81, 36-44 (2013)

4. Zhao, P, Balakrishnan, $\mathrm{N}$ : On the right spread ordering of parallel systems with two heterogeneous components. Statistics 48, 447-455 (2014)

5. Khaledi, B, Kochar, SC: Some new results on stochastic comparisons of parallel systems. J. Appl. Probab. 37, 1123-1128 (2000)

6. Khaledi, B, Kochar, SC: Weibull distribution: some stochastic comparisons results. J. Stat. Plan. Inference 136 3121-3129 (2006)

7. Dykstra, R, Kochar, SC, Rojo, J: Stochastic comparisons of parallel systems of heterogeneous exponential components. J. Stat. Plan. Inference 65, 203-211 (1997)

8. Rojo, J, He, GZ: New properties and characterizations of the dispersive ordering. Stat. Probab. Lett. 11, 365-372 (1991)

9. Kochar, SC, Ma, CS: Dispersive ordering of convolutions of exponential random variables. Stat. Probab. Lett. 43, 321-324 (1999)

10. Kochar, SC, Xu, M: Comparisons of parallel systems according to the convex transform order. J. Appl. Probab. 46 342-352 (2009)

11. Fang, L, Zhang, X: Stochastic comparisons of series systems with heterogeneous Weibull components. Stat. Probab. Lett. 83, 1649-1653 (2013)

12. Fang, L, Zhang, X: New results on stochastic comparison of order statistics from heterogeneous Weibull populations. J. Korean Stat. Soc. 41, 13-16 (2012)

13. Prabhakar Murthy, DN, Xie, M, Jiang, R: Weibull Models. Wiley, New York (2004)

14. Shaked, M, Shanthikumar, JG: Stochastic Orders and Their Applications. Academic Press, San Diego (1994)

15. Marshall, AW, Olkin, I: Inequalities: Theory of Majorization and Its Applications. Academic Press, New York (1979)

16. Saunders, IW, Moran, PA: On the quantiles of the gamma and F distributions. J. Appl. Probab. 15, 426-432 (1978)

17. Kochar, SC, Xu, M: On the right spread order of convolutions of heterogeneous exponential random variables. J. Multivar. Anal. 101, 165-176 (2010) 